$$
\text { "tmcs-kiraly-int" — 2011/11/20 — 19:42 — page } 241 \text { — \# }
$$

\title{
Teaching integral transforms in secondary schools
}

\author{
SÁNDOR KIRÁLY
}

\begin{abstract}
Today, Hungarian students in the secondary schools do not know the idea of complex numbers, and they can not integrate except those ones who learn mathematics in advance level. Without this knowledge we can teach Fourier transform for students. Why should we teach Fourier transform (FT) or Wavelet transform (WT) for them? To teach image file formats like JPEG, (JPEG2000) we need to talk about integral transforms. For students who are good in computer programming, writing the program of $1 \mathrm{D} \mathrm{FT}$ or $2 \mathrm{D} \mathrm{FT}$ is a nice task. In this article we demonstrate how we can teach Fourier and Wavelet transform for students in secondary school.
\end{abstract}

Key words and phrases: Fourier transform, Wavelet transform, magnitude, time domain, frequency domain, spatial domain, DC.

ZDM Subject Classification: B50, C70, D40, D50, F50, F90, U70.

\section{Introduction}

The base of understanding the point of FT is understanding the 1D FT. That is why we use not only a lot of figures but an Excel spreadsheet for teaching this topic. Using Excel spreadsheet, students can create signals and their spectra and they can do the FT on their own data. The formula of FT should be presented at the end of the topic, or we can omit it. But if we want to make students implement the FT we need to use and explain the formula. For several students it will be the first time that they meet the idea of integration and complex numbers. It can not be our goal to review this topic completely. Students will learn this later at another level of education.

Copyright (C) 2011 by University of Debrecen 


$$
\text { "tmcs-kiraly-int" — 2011/11/20 — 19:42 — page } 242 \text { — \#2 }
$$

Then we do the discrete FT on a sampled sign and we calculate the Fourier coefficients at different frequencies. If students really understand how the $1 \mathrm{D}$ FT works, then we can go on teaching the $2 \mathrm{D}$ FT used for image processing. We teach only the discrete version, because it is used in image processing. We show a lot of images as the best way to become familiar with FT is to see lots of images and lots of their FTs. After being acquainted with FT the teaching of Wavelet transform is much easier if we use the attained ideas of FT and emphasize the differences and the advantages of WT.

\section{Where do we use FT?}

For students this topic is very difficult and hard to understand. Accordingly, during the teaching it is very important to maintain the students' motivation and keep to the principles of didactics of teaching maths mainly the principle of expressity, the principle of spiral and the principle of operation. [1] Before teaching Fourier transform (FT) and Wavelet transform (WT), we can ask student to search themes of the history of Fourier transform, the major works of JeanBaptiste Joseph Fourier and the applications of FT and WT as homework on the Internet. This may pique their interest as the dispute of Lagrange and Laplace, lost for ages, is really interesting and the applications of transforms and its versions (iPod/MP3 players, DVD players, 4G mobiles, 801.11g Wi-Fi instruments, data compression, noise reduction, earthquake monitoring) will surely maintain the student's interest. After this we can teach them what the signal and the spectrum are.

\section{Signals and spectra}

First we need to define the meaning of transform. As students know the idea of function we can start here. A function assigns exactly one value to each input value. For example: $y=2 x+1$ is a function, where $x$ is a value in the input quantity, and $y$ is a value of the function according to $x$. It multiples the value of $x$ by two and adds one to the product and returns the result $y$. There are functions with more arguments that calculate a value by using more than one value. For example: $y=2 x_{1}+3 x_{2}+4 x_{3}$ is a function that returns with the value of $y$ by having the input arguments $x_{1}, x_{2}, x_{3}$. The transform can be imagined as function but it is a mapping of the input quantity to the output one by a 
given function. There are several transforms but Fourier transform is probably the most popular.

After the introduction of transform we can introduce the idea of FT by using the inductive method. First, we show figures of signals and their spectra. Figure 1 presents a sine wave in time which represents a signal. The periodic signal in Figure 1 passing through the horizontal axis 7 times (including the starting value).

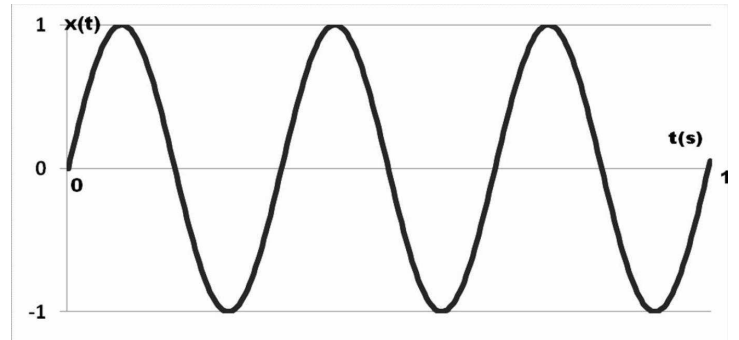

Figure 1. Sin wave of $3 \mathrm{~Hz}$. In the $x$-axis, there is the time interval, $t(s)$, values given in seconds, while the $y$-axis represents the values of the signal $x(t)$

"An analogue signal is any continuous signal for which the time varying feature (variable) of the signal is a representation of some other time varying quantity, for example analogous to another time varying signal." [8]

In Figures 2 and 3, we can see a sine wave of $10 \mathrm{~Hz}$ and $50 \mathrm{~Hz}$, respectively. Make us analyze these signals with our students. Point out the differences of plots, mainly to the point that signals with higher frequency change faster and that the frequency of these signals is constant.

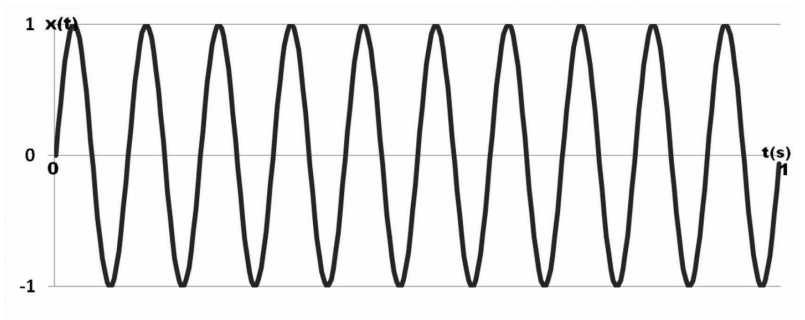

Figure 2. Sin wave of $10 \mathrm{~Hz}$
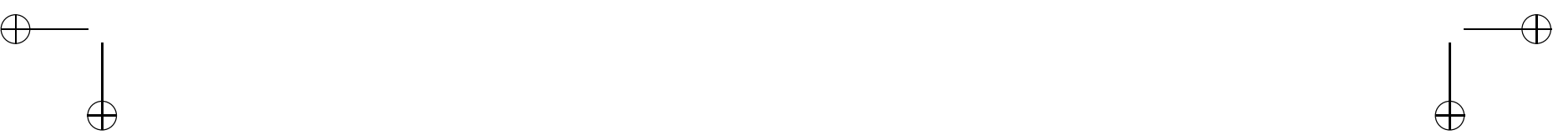


$$
\text { "tmcs-kiraly-int" — 2011/11/20 — 19:42 — page } 244 \text { — \# }
$$

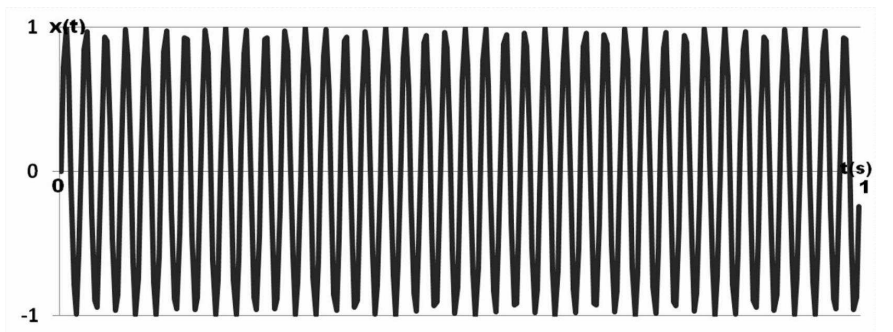

Figure 3. Sine wave of $50 \mathrm{~Hz}$

These signals are time-domain ones. A time-domain signal is a function of time. In other words, when we plot the signal one of the axes is time (independent variable), and the other one (i.e., the dependent variable) is the value of a function at a current time. When we plot time-domain signals, we obtain a time-amplitude representation of the signal. If the students understand this, then we can explain the idea of spectrum.

It is not always the best representation of the signal for most signal processing related applications. In many cases, the most distinguished information is hidden in the frequency content of the signal.

Spectrum is a range of frequencies in which the desired signal will be transmitted. [6] The frequency spectrum of a signal shows what frequencies exist in the signal. Figure 4 shows the spectra of the signals presented in Figures 1-3.

Since the signals in Figures 1-3 are composed of sine waves with only one frequency so their spectra are quite simple. (In continuous case it should be a Dirac-delta function.)

For every frequency in $x$-axis we have an amplitude value. In Figure 4(a), the zero magnitude belongs to each frequency except $3 \mathrm{~Hz}$. It means only one frequency can be found in the signal. In Figure 4(b) and in Figure 4(c), the signal is composed of sine waves with frequency $10 \mathrm{~Hz}$ and $50 \mathrm{~Hz}$, respectively, hence a peak appears at $10 \mathrm{~Hz}$ and $50 \mathrm{~Hz}$ in the frequency domain, respectively.

Tell students that the spectrum of a real signal is usually symmetric. However, since the symmetric part is exactly a mirror image of the first part, it provides no additional information, and therefore, this symmetric second part is usually not shown. That is why we do not present it.

Then show students signals composed of sine waves with different frequencies and amplitudes. The signal in Figure 5(a) has three frequency components $(3 \mathrm{~Hz}$, 


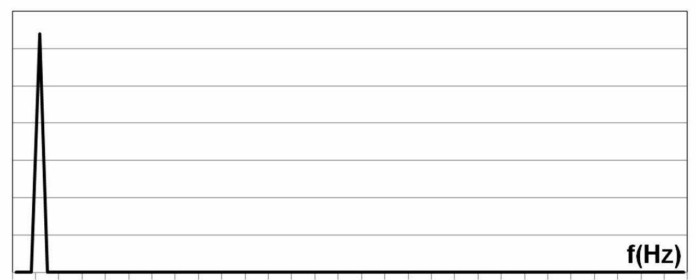

$\begin{array}{llllllllllllllllll}0 & 5 & 10 & 15 & 20 & 25 & 30 & 35 & 40 & 45 & 50 & 55 & 60 & 65 & 70 & 75 & 80 & 85\end{array}$

(a) Spectrum of sine wave of $3 \mathrm{~Hz}$

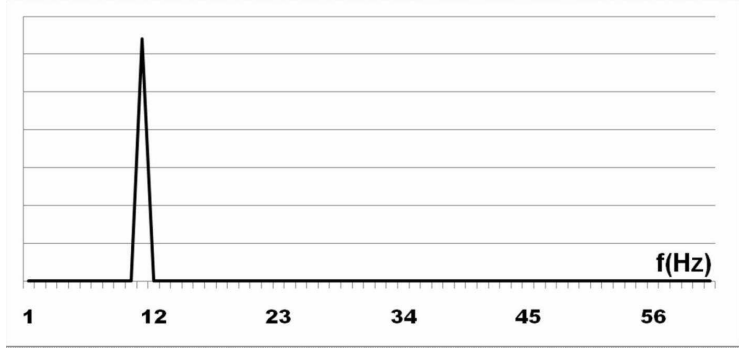

(b) Spectrum of sine wave of $10 \mathrm{~Hz}$

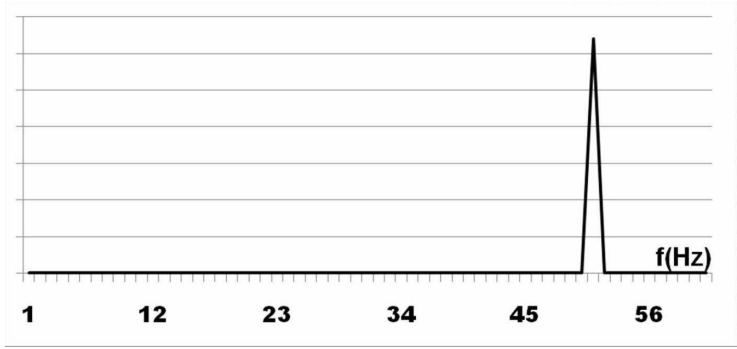

(c) Spectrum of sine wave of $50 \mathrm{~Hz}$

Figure 4

$10 \mathrm{~Hz}$, and $20 \mathrm{~Hz}$ ), but without knowing the formula or the spectrum of the signal it would be hard to determine them.

Point out and give examples for students why we need to define the spectrum of signals. The Nyquist-Shannon sampling theorem states that perfect reconstruction of a signal is possible when the sampling frequency is greater than twice the maximum frequency of the signal being sampled, or equivalently, when the Nyquist frequency (half the sample rate) exceeds the highest frequency of the 


$$
\text { "tmcs-kiraly-int" — 2011/11/20 — 19:42 — page } 246 \text { — \#6 }
$$

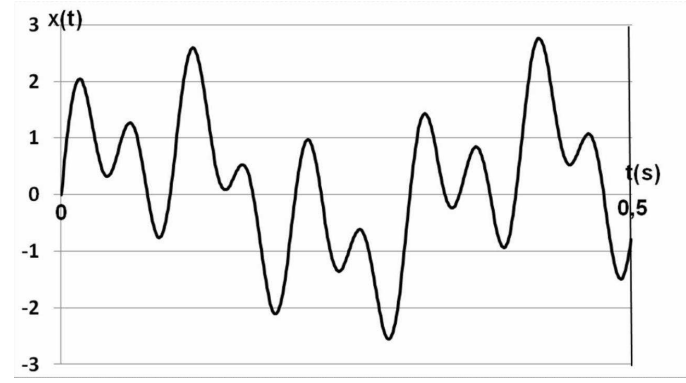

(a)

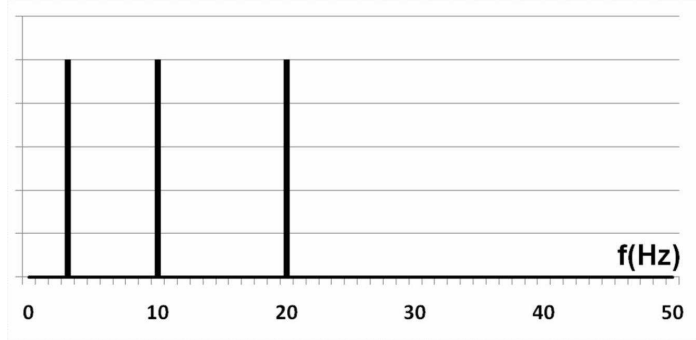

(b)

Figure 5. $\sin (2 \pi \cdot t \cdot 3)+\sin (2 \pi \cdot t \cdot 10)+\sin (2 \pi \cdot t \cdot 20)$ in (a) and its spectrum in (b)

signal being sampled. $\left(f_{c}=\frac{1}{2 \Delta t}\right)$ If lower sampling rates are used, the original signal's information may not be completely recoverable from the sampled signal. [7] In order to this we need to know the highest frequency in the signal.

\section{Fourier transform}

The question is: how can we define the spectrum of a signal? And how can we prepare figures shown earlier? The answer is Fourier transform. Using the following figure we can explain students what FT does.

The FT transforms the signal in the time-domain into the frequency-magnitude domain. If we have $N$ real values of amplitudes in the time-domain after FT, then we will have $N$ complex values of amplitudes in the frequency. FT transforms a signal from time domain to the frequency domain. The inverse FT transforms a signal from frequency domain to the time domain. 


$$
\text { "tmcs-kiraly-int" — 2011/11/20 — 19:42 — page } 247 \text { — } 7
$$

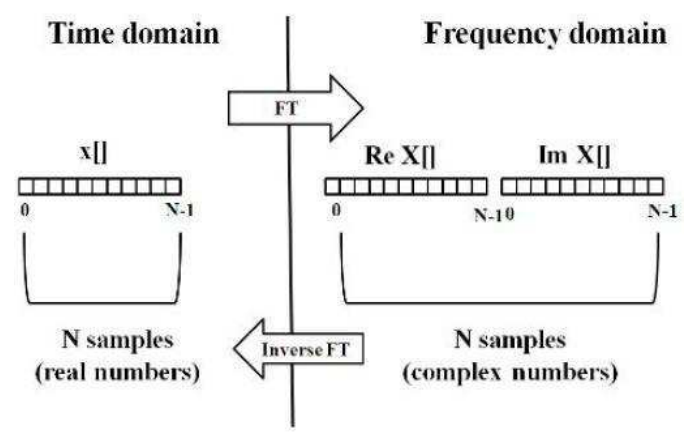

Figure 6. Illustrating the Fourier transform and the inverse FT

\subsection{Fourier transform using Excel}

An old saying demonstrates one of the principles of mathematics-didactics: "What I am hearing I'll forget, what I see I'll memorize and what I am doing I'll understand." The previous figures can be made by students. Using Excel, they can represent signals and their spectra. [11] First we have to make the suitable spreadsheet then students need to change the frequencies of signals or the formula of the signals only. Before creating the spreadsheet, we have to check whether we can see the Data Analysis command (in Data tab). If we can not, we need to install Analysis ToolPak in Microsoft Excel. To install the Analysis ToolPak go to the Office button, click Excel settings, Add-Ins and select the Analysis ToolPak. In the following we give some instructions by an example. Label five columns in Excel as follows: Time, Data, FFT frequency, FFT magnitude, and FFT complex. This will correspond to columns A to E in the Excel spreadsheet. As Excel uses FFT for calculating Fourier coefficients the number of data should be power of 2. (It could be any number of data but Excel requires the power of 2.) Type this number in cell H3. In H4 type the time period. It is 1 second now. Then calculate the time interval in $\mathrm{H} 5$ which is $\mathrm{H} 4 / \mathrm{H} 3$. In cell A2 type 0 then fill the cells below according to the following formula: in cell A3 type $=\mathrm{A} 2+\mathrm{H} \$ 5$, in cell A4 type $\mathrm{A} 3+\mathrm{H} \$ 5$, etc. or click and drag until cell A1025. In column B we type data by using a formula. In cell B2 type 0 then in cell B3 type formula $=\mathrm{SIN}\left(2 * \mathrm{PI}()^{*} \mathrm{~A} 3{ }^{*} \mathrm{G} \$ 1\right)$ and copy this formula to the other ones until B1025. In cell G1, there will be the frequency value. In the previous examples it was 3, 10 and 50. So in column A there are the time values in column B there is data: a sine wave. Retyping G1 students can change the frequency value. To represents 


$$
\text { "tmcs-kiraly-int" — 2011/11/20 — 19:42 — page } 248 \text { — } 8
$$

the signal in time, we just need to mark the column A and column B and to insert a line chart. $x$-axis represents the time values $y$-axis represents the amplitudes of the signal. We will get a chart similar to Figure 1. Changing the value in G1 we can follow the changing of the frequency of the signal. Furthermore, if we change the formula of the signal typed in column B then the chart will be changed immediately.

\begin{tabular}{|c|c|c|c|c|c|}
\hline \multicolumn{2}{|r|}{ B3 } & $\cdot(3$ & \multicolumn{3}{|c|}{$=\operatorname{SIN}\left(2^{*} P 1(1) * A 3^{*} G \$ 1\right)$} \\
\hline 4 & A & B & c & D & E \\
\hline 1 & Time & Data & FFT frequency & FFT Magnitude & FFT complex \\
\hline 2 & 0 & 0 & 0 & 0,059441032 & 20,951056516295146 \\
\hline 3 & 0,00098 & 0,061320736 & 1 & 0,06189692 & $=0,986666039968371-8,53502736963364 \mathrm{E}-002 \mathrm{i}$ \\
\hline 4 & 0,00195 & 0,122410675 & 2 & 0,070013552 & $1,10518220267952-0,182915419211132 \mathrm{i}$ \\
\hline 5 & 0,00293 & 0,183039888 & 3 & 0,086767531 & $1,352646544634-0,312522110499678 \mathrm{i}$ \\
\hline 6 & 0,00391 & 0,24298018 & 4 & 0,121164421 & $1,86702365152152-0,522026627593649 \mathrm{i}$ \\
\hline 7 & 0,00488 & 0,302005949 & 5 & 0,20908465 & $5,19516598457557-0,991115708979158 \mathrm{i}$ \\
\hline 8 & 0,00586 & 0,359895037 & 6 & 0,74783921 & $111,3835920434614-3,68582205764904 \mathrm{i}$ \\
\hline 9 & 0,00684 & 0,41642956 & 7 & 0,515163647 & $7-7,84470300692435+2,53009726146858 \mathrm{i}$ \\
\hline
\end{tabular}

Figure 7. The part of the Excel table with FFT complex and FFT magnitude

However, our goal is representing not only the time domain but the spectrum of a signal as well. So we need to complete the column E. For this we need to use Data/Data analysing/Fourier analysis and to enter the input range of data. In our example we have 1024 data point $\left(2^{10}\right)$ in column B. Therefore, input range is the data located at $\$ \mathrm{~B} \$ 2: \$ \mathrm{~B} \$ 1025$. Then we need to select the output range for FFT complex number generated by Fourier Analysis to be located at $\$ \mathrm{E} \$ 2: \$ \mathrm{E} \$ 1025$. Calculate the FFT magnitude (column D) by finding the absolute value of the FFT complex of column E and multiply it by $2 /$ number of data. In our example, it is 1024 . So in cell D2 type the following formula: $=2 / 1024 * \operatorname{IMABS}(\mathrm{E} 2)$. Then drag the formula in D2 down to D1025. In column C we type the frequency values. In cell $\mathrm{C} 2$ type 0 , from cell $\mathrm{C} 3$ type the following formula: $=\mathrm{C} 2+\mathrm{H} \$ 3$. $\mathrm{H} 6=\mathrm{H} 3: \mathrm{H} 5$. In cell $\mathrm{H} 6$ there is the sampling frequency $\left(\Delta f=\frac{1}{N \cdot \Delta t}\right)$, in cell H3 there is the number of data. Plotting them, $x$-axis represents the frequency values (column C) $y$-axis represents the magnitude values (column D). If we plot the whole area (D2:D1025), we can realize that the spectrum of signals will be symmetric. If we have a $10 \mathrm{~Hz}$ signal we can see a line at 10 and 1014 too. If we want to avoid it, plot only the half of samples (or less). What can we use our spreadsheet for? We can generate sine or cosine waves, we can plot them, we can change their frequencies and we can plot their spectrums. If we change the frequency of a signal or if we generate another signal we have to calculate the FT 


$$
\text { "tmcs-kiraly-int" — 2011/11/20 — 19:42 — page } 249 \text { — \#9 }
$$

coefficients again (Data/Data_analysis/Fourier_Analysis). By using this spreadsheet we can demonstrate some attributes of FT such as linearity or scaling.

Checking our spreadsheet (see Figure 7) students can see that FT transforms from $N$ real numbers to $N$ complex numbers. Complex numbers consist of real and imaginary parts. Plotting the spectrum we need only the magnitude which is a kind of amplitude. (We can explain the meaning of magnitude but later it is better.)

\subsection{How does FT work?}

Up to now students will may imagine what FT does but they will not understand how it works. For the explanation we use the formula of FT. It decomposes a signal to complex exponential functions of different frequencies. The way it does this, is defined by the following two equations:

$$
\begin{aligned}
& X(f)=\int_{-\infty}^{+\infty} x(t) \cdot e^{-2 \pi \cdot i \cdot f \cdot t} d t \\
& x(t)=\int_{-\infty}^{+\infty} X(f) \cdot e^{2 \pi \cdot i \cdot f \cdot t} d f
\end{aligned}
$$

In the above equation, $t$ stands for time, $f$ stands for frequency, and $x$ denotes the signal. $i=\sqrt{-1}$ is a complex number. A complex number is a number consisting of a real and imaginary part. It can be written in the form $a+b i$, where $a$ and $b$ are real numbers, and $i$ is the standard imaginary unit with the property $i^{2}=-1$. [13] [2] The complex numbers contain the ordinary real numbers, but extend them by adding in extra numbers and correspondingly expanding the understanding of addition and multiplication.

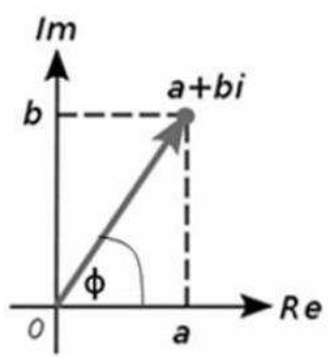

Figure 8. A complex number can be visually represented as a pair of numbers forming a vector on a diagram 


$$
\text { "tmcs-kiraly-int" — 2011/11/20 — 19:42 — page } 250 \text { — \#10 }
$$

Note that $x$ denotes the signal in time domain and the $X$ denotes the signal in frequency domain. This convention is used to distinguish the two representations of the signal. Equation (1) is called the Fourier transform of $x(t)$, and equation (2) is called the inverse Fourier transform of $X(f)$, that is $x(t)$. We need to emphasize to students that FT is an inverse transform. According to the formula the signal $x(t)$ is multiplied by an exponential term, at some certain frequency $f$, then it integrated over all times. The equation (1)-(2) can also be written as:

$$
\begin{aligned}
& X(f)=\int_{-\infty}^{+\infty} x(t) \cdot(\cos (2 \pi \cdot f \cdot t)-i \sin (2 \pi \cdot f \cdot t)) d t \\
& x(t)=\int_{-\infty}^{+\infty} X(f) \cdot(\cos (2 \pi \cdot f \cdot t)+i \sin (2 \pi \cdot f \cdot t)) d f
\end{aligned}
$$

$\left(\right.$ as $\left.e^{i t}=\cos (t)+i \sin (t)\right)$

This equation shows better how FT works. FT decomposes the signals to sine and cosine functions. It generates the signal as the integral of sine and cosine functions. So the base functions of $\mathrm{FT}$ are $\cos (2 \pi \cdot f t)$ (real part) and $i \sin (2 \pi \cdot f t)$ (imaginary part). FT multiplies the original signal by a complex expression which has sines and cosines of frequency $f$. Then it integrates this product. If the result of this integration (which is nothing but some sort of infinite summation, the FT coefficient) is a relatively large value, we say that: the signal $x(t)$ has a dominant spectral component at frequency $f$. This means that a major portion of this signal is composed of frequency $f$. If the integration result is a small value, it means that the signal does not have a major frequency component of $f$ in it. If this integration result is zero, then the signal does not contain the frequency $f$ at all. The following figures can make the explanation much understandable. [5]

In Figure $9(\mathrm{a})$ there is a signal $(x(t))$. Figure $9(\mathrm{~b})$ shows a function: $\cos (2 \pi \cdot 1 \cdot t)$. Figure $9(\mathrm{c})$ shows the result of the multiplication and the integration. $\int_{-\infty}^{+\infty} x(t) \cdot \cos (2 \pi \cdot 1 \cdot t) d t$. According to that the signal has no frequency at $1 \mathrm{~Hz}$, as the area (the result of integration) is zero.

Figure 10(a) shows the original signal, and below there is $\cos (2 \pi \cdot 0.12 \cdot t)$. Figure $10(\mathrm{c})$ shows the result of the integration. The area is almost one so the signal contains this frequency component (0.12). (The two signals are very similar.)

As computers can not work continuous signals we need a formula that can give the Fourier transform of finite and discrete signals. This is the discrete Fourier transform (DFT) that helps us to show students more graphically how 


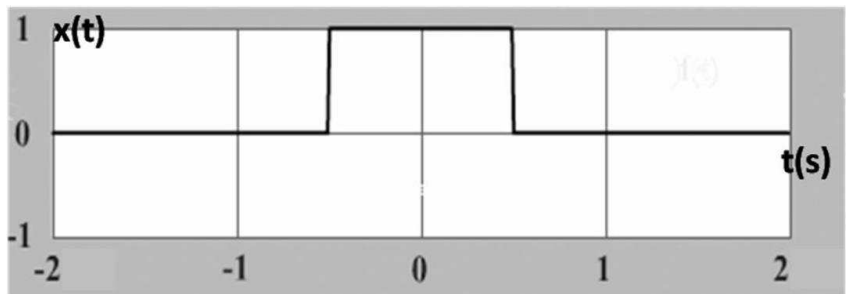

(a)

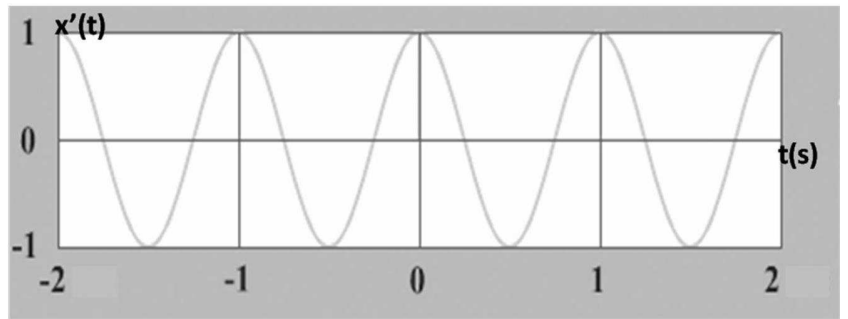

(b)

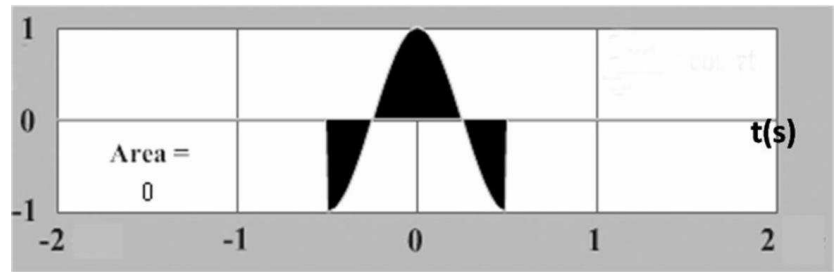

(c)

Figure 9. There is a signal in (a), $\cos (2 \pi \cdot 1 \cdot t)$ is in (b) and the result of the multiplication and the integration of them in (c)

the Fourier transform works. The equation of DFT is:

$$
\begin{gathered}
X\left(f_{k}\right)=\frac{1}{N} \sum_{j=0}^{N-1} x\left(t_{j}\right)\left(\cos \left(2 \pi \cdot f_{k} \cdot \frac{t_{j}}{N}\right)-i \sin \left(2 \pi \cdot f_{k} \cdot \frac{t_{j}}{N}\right)\right), t_{j}=j \cdot \Delta t \\
\Delta f=\frac{1}{N \cdot \Delta t}, \quad f_{k}=\Delta f \cdot k, \quad \text { where } k=1, \ldots, N
\end{gathered}
$$

Explain the differences to students. We have to sample the original signal so we have $N$ samples. As it is finite, we replace the integral symbol by a sum. For a given frequency $f_{k}$ we multiply the signal (the sample values) by an expression and the value got we sum. This will be the Fourier coefficient of the signal at 


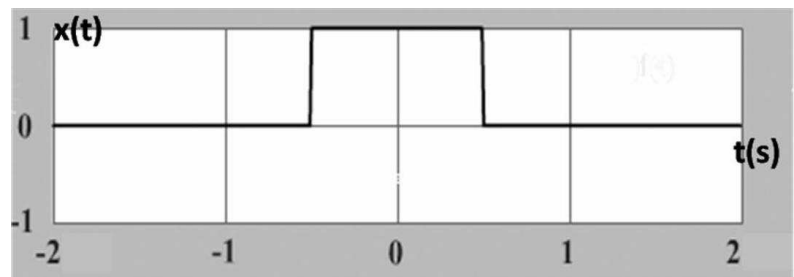

(a)

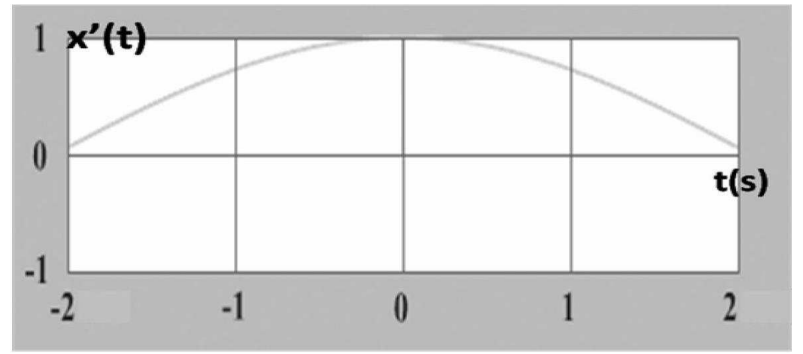

(b)

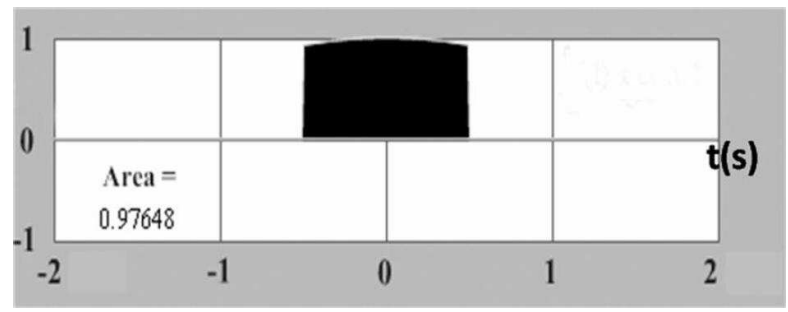

(c)

Figure 10. There is a signal in (a), $\cos (2 \pi \cdot 0.12 \cdot t)$ is in (b) and the result of the multiplication and the integration of them in (c)

frequency $f_{k}$. If this coefficient is much greater than zero, it means that this signal has a major frequency component of $f_{k}$. FT does this operation for each frequency until $N-1$. Show a real example to students. Figure 11 shows the sampled signal. The amplitude values are the following (approximately): $x_{0}=-0.44 ; x_{1}=0.45$; $x_{2}=0.55 ; x_{3}=0.71 ; x_{4}=0.73 . x_{5}=0.72 ; x_{6}=0.74 ; x_{7}=0.71 ; x_{8}=0.27$; $x_{9}=-0.58 ; x_{10}=-0.81 ; x_{11}=-0.13 ; x_{12}=0.73 ; x_{13}=0.35 ; x_{14}=-0.61$; $x_{15}=-1.00$. 


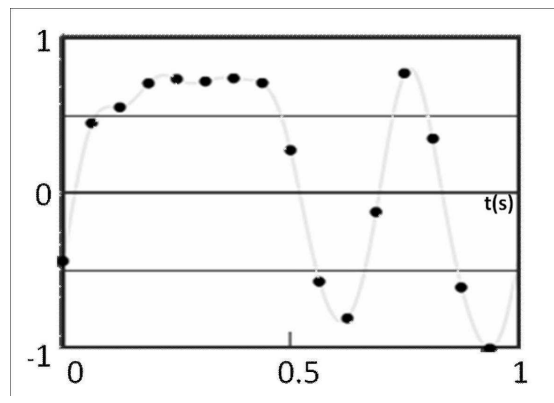

Figure 11. A sampled signal

We need to calculate the Fourier coefficients of this signal by using the equation of DFT at frequency $f_{0}=0, f_{1}=1$ and $f_{2}=2$ :

$$
\begin{aligned}
& x_{0}=\frac{1}{16} \sum_{j=0}^{15} x\left(t_{j}\right)\left(\cos \left(2 \pi \cdot 0 \cdot \frac{t_{j}}{16}\right)-i \sin \left(2 \pi \cdot 0 \cdot \frac{t_{j}}{16}\right)\right) \\
& x_{1}=\frac{1}{16} \sum_{j=0}^{15} x\left(t_{j}\right)\left(\cos \left(2 \pi \cdot 1 \cdot \frac{t_{j}}{16}\right)-i \sin \left(2 \pi \cdot 1 \cdot \frac{t_{j}}{16}\right)\right) \\
& x_{2}=\frac{1}{16} \sum_{j=0}^{15} x\left(t_{j}\right)\left(\cos \left(2 \pi \cdot 2 \cdot \frac{t_{j}}{16}\right)-i \sin \left(2 \pi \cdot 2 \cdot \frac{t_{j}}{16}\right)\right)
\end{aligned}
$$

The coefficients are: $x_{0}=2.44+0 i ; x_{1}=-1.16-4.04 i ; x_{2}=-3.14+0.62 i$;

DFT replaced 16 real numbers given in the time domain by 16 complex values in the frequency domain. Unfortunately students are not familiar to it . Remember them that in Excel we used magnitude values instead of FFT coefficients. It is time to give the formula of the magnitude: $|z|=\sqrt{R e^{2}+I^{2}}$.

In our example above for frequency 0 we got $2.44+0 i$ complex number whose magnitude value is $\sqrt{2,44^{2}+0^{2}}=2.44$. For $x_{1}$ it is $\sqrt{-1,16^{2}+4,040^{2}}=4.2$.

\subsection{Two-dimensional DFT}

2D DFT used mainly in digital image processing. While 1D DFT transforms the signal from time domain into the frequency domain, 2D DFT transforms images from the 2D spatial domain into the frequency domain. Explain the 


$$
\text { "tmcs-kiraly-int" — 2011/11/20 — 19:42 — page } 254 \text { — \#14 }
$$

importance of this transform to students: image analysis, noise filtering and compressing of images are much easier in the frequency domain than in the spatial domain. The question is: what is the frequency domain of an image?

The frequency domain is a $2 \mathrm{D}$ space in which each image value at image position $F$ represents the amount that the intensity values in image I vary from a specific distance related to $F$. In the frequency domain, changes at an image position correspond to changes in the spatial frequency. For example, suppose that there is the value 20 at the point that represents the frequency 0.1 (or 1 period every 10 pixels). This means that in the corresponding spatial domain image I, the intensity values, vary from dark to light and back to dark over a distance of 10 pixels, and that the contrast between the lightest and darkest is 40 gray levels (2 times 20). [15]

In most of cases, the Fourier transform is used to convert images from the spatial domain into the frequency domain and vice-versa. The extension of the Fourier Transform to 2D is pretty simple. First we take the 1D FT of every row of the image, then on this result we take the 1D FT of every column. For an image of size $M \times N$, the two-dimensional DFT is given by:

$$
F(u, v)=\frac{1}{N \cdot M} \sum_{k=0}^{M-1} \sum_{l=0}^{N-1} f\left(x_{k}, y_{l}\right) e^{-2 \pi i\left(\frac{u x_{k}}{M}+\frac{v y_{l}}{N}\right)}
$$

It can be expressed the following way:

$$
F(u, v)=\frac{1}{N} \sum_{l=0}^{N-1} P\left(u, y_{l}\right) e^{-2 \pi i \frac{v y_{l}}{N}}
$$

where

$$
P(u, y)=\frac{1}{M} \sum_{k=0}^{M-1} f\left(x_{k}, y\right) e^{-2 \pi i \frac{u x_{k}}{M}}
$$

The following figures (Figure 12 and Figure 13) show special images and their FT. [4] Show them to students and explain them. As the transform results are complex numbers so for displaying the image we use only the magnitude values. Magnitude tells "how much" of a certain frequency component is presented in the image. The Fourier images are shifted in such a way that the DC-value (the average brightness of the image) $F(0,0)$ is displayed in the center of the image. The further away from the center an image point is, the higher its corresponding frequency is. [12]

The FT for each just has a single component, represented by two bright spots symmetrically placed about the center of the FT image (see Figure 12(b)). 


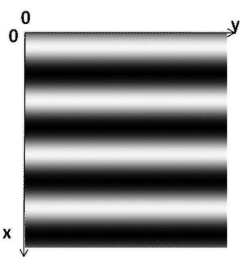

(a)

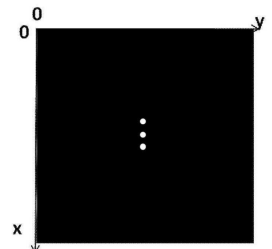

(b)

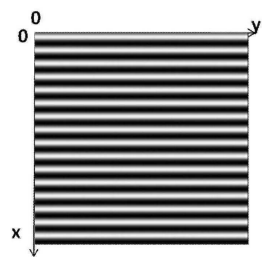

(c)

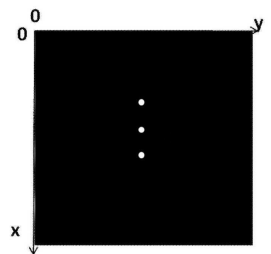

(d)

Figure 12. Images (a) (c) and their FT (b) (d)

In the Figure 12(c) there is a sine function with a higher frequency is used to generate the image, so the two dots are further away from the origin to represent the higher frequency. If students examine it carefully, they can see that both FT image contains 3 main values: the DC-value and, since the Fourier image is symmetrical to its center, two points corresponding to the frequency of the stripes in the original image. The two points lie on a vertical line through the image center, because the image intensity in the spatial domain changes the most if we go along it vertically.

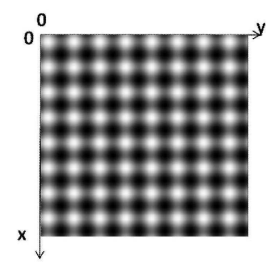

(a)

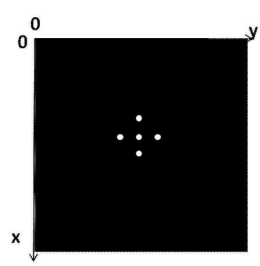

(b)

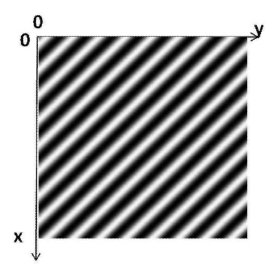

(c)

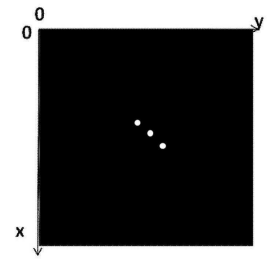

(d)

Figure 13. Images (a) (c) and their FT (b) (d)

If we rotate the image, the spectrum will be rotated in the same direction (see Figure 13(c) 13(d)). Figure 13(a) shows the sum of two sine functions. The previous figures demonstrate the working of $2 \mathrm{D}$ DFT well, but we need to show more similar images to students. The following two figures (Figure 14 and Figure 15) demonstrate what we use 2D DFT in practise for. [10]

We transformed the original images into the frequency domain where we filtered the low frequencies by using a high-pass filter, so we got a sharper image after performing the inverse DFT. Image sharpening requires a "sharpening" filter 


$$
\text { "tmcs-kiraly-int" — 2011/11/20 — 19:42 — page } 256 \text { — \#16 }
$$

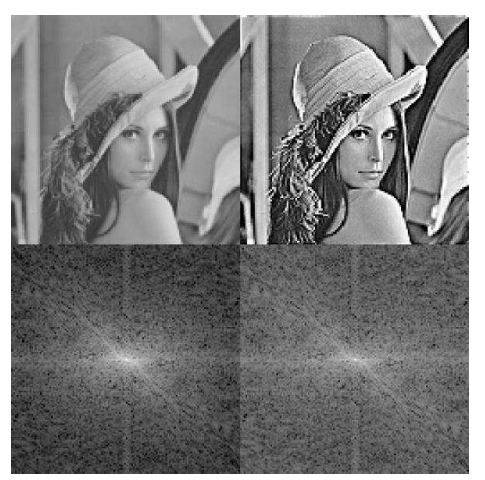

Figure 14. The original image with its FT and the sharpened image with its FT

or high frequency emphasis filter as high frequencies in frequency domain mean the edges and the noise in the image. This type of filter preserves some of the low frequency information but relatively boosts the higher frequencies. To do such a thing, we construct our own filter which is piecewise-linear. The filter is circularly symmetrical and will have coefficients as follows:

$$
\begin{array}{cr}
0 & 0.5 \\
96 & 4.0 \\
127 & 4.0
\end{array}
$$

In other words, Fourier coefficients of frequency-distance 0 from the origin are multiplied by 0.5. As we go away from the origin or zero frequency, out to frequency-distance 96 , the multiplier is interpolated between 0.5 and 4.0. From then outward, the multiplier is 4.0. So higher frequency coefficients are multiplied by values greater than 1.0 and lower frequency coefficients are multiplied by values less than 1.0. The overall net effect on the image power is that it is unchanged. $[10]$

We can reduce the image noise if we use a low-pass filter, because noise consists largely of high frequencies which are attenuated by a low-pass filter. Figure 15 shows an image with noise (a), the result of applying the low-pass filter is in Figure 15(b) and the filter is in Figure 15(c). 


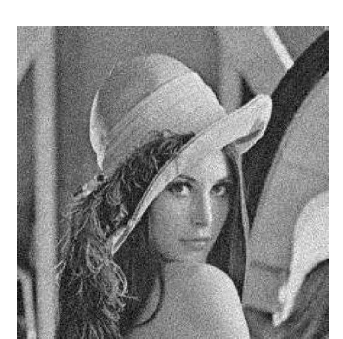

(a)

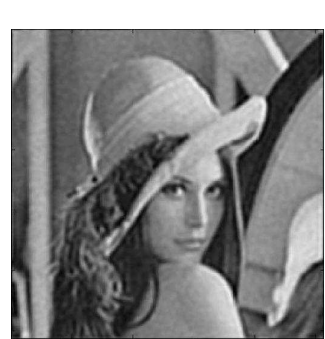

(b)

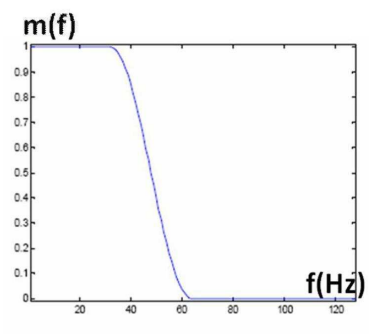

(c)

Figure 15. The noisy image in (a), the filtered image in (b) and the used filter in (c)

\section{Wavelet transform}

Unfortunately FT does not give time and frequency values of the signal at the same time as opposed to the Wavelet transform. WT can transform from time domain into time-frequency domain. The teaching of WT should begin with comparing FT with WT as students have already known FT. The main difference between them is their base functions. As opposed to FT the base functions of WT are not sine or cosine waves but wavelets (small waves). These are the compressed and stretched versions of the base wavelet (named mother wavelet). Wavelets are not predefined. The key to the success of the transform is the selection of the adequate mother wavelet. Show some well-known wavelets to students such as Haar or Gauss. [14]

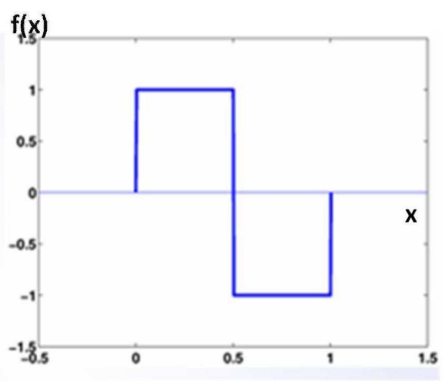

(a) Haar wavelet

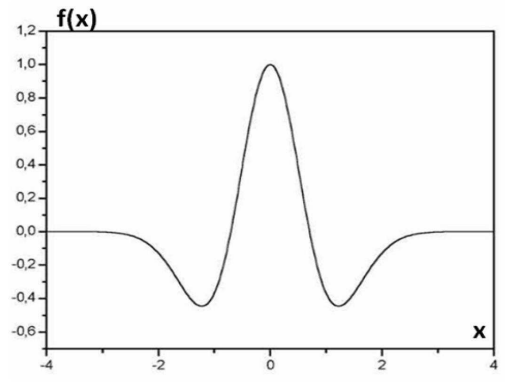

(b) Gauss wavelet

Figure 16 


$$
\text { "tmcs-kiraly-int" — 2011/11/20 — 19:42 — page } 258 \text { — \#18 }
$$

The meaning of this transform is that we dilate and compress the mother wavelet and shift them along the signal then, as we did it in FT, we multiply the wavelet (at the current scale value) by the signal. We check the similarity between the wavelets and the signal at the current scale parameter. (The measure of compressing is the scale parameter and defined as 1 /frequency.) 1 /frequency is the wave-length or period-length of a periodic signal. [3] So the Wavelet analysis is also a similarity measure between the base functions and the signal such as FT. The base of the similarity is also the similar frequency content. The calculated coefficients give information to us whether the wavelet at the current scale is similar to the signal or not. If the signal has a spectral component that corresponds to the current value of scale parameter, the product of the wavelet with the signal at the location, where this spectral component exists, gives a relatively large value. If the spectral component that corresponds to the current value of the scale is not presented in the signal, the product value will be relatively small or zero.

The computation of CWT is the following:

(1) First choose the suitable wavelet function and place the wavelet at the beginning of the signal at the point which corresponds to $t=0$ and compare it with the signal at scale one. (see Figure 17(a))

(2) The wavelet function at scale one is multiplied by the signal and then integrate over all times. Then the result of the integration is multiplied by the constant number $\frac{1}{\sqrt{s}}$. The calculated value (c) shows the similarity between the signal and the wavelet in this part of the signal. (It depends on the picked mother wavelet.)

(3) Shift the wavelet to the right and repeat steps 1 and 2 until we have covered the whole signal. (see Figure 17(b))

(4) Scale (stretch) the wavelet and repeat steps 1 through 3. (see Figure 17(c))

(5) Repeat steps through 1 through 4 for all scales.

\section{Summary}

The main aim of this article was to give ideas of teaching integral transforms, mainly FT and WT for students in secondary school. We presumed that students know the idea of frequency, amplitude, and they know sine and cosine functions. In this paper first, we suggest how we can use MS Excel for representing signals in time domain and for representing their spectra. Using this spreadsheet we can 


$$
\text { "tmcs-kiraly-int" — 2011/11/20 — 19:42 — page } 259 \text { — \#19 }
$$

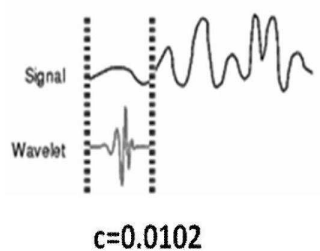

(a)

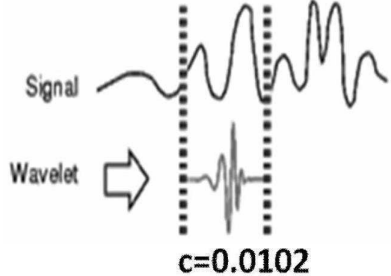

(b)

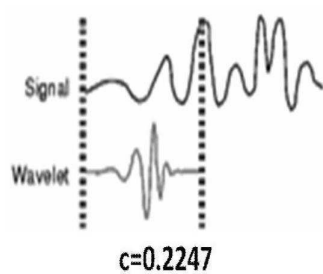

(c)

Figure 17. The signal and the wavelet is in (a), we move the wavelet in (b), we compress the wavelet and repeat steps in (c)

represent any signal with its spectrum. Demonstrating the continuous FT this article can help teachers to make an integral transform clear for students. We could use other softwares (FreeMat, Octave, Scilab, LabView) [9] but Excel is available in most secondary schools, we do not have to download and install, and students know this software very well.

Then, by using figures, this article presents how FT breaks up the sign as sum of sine waves and helps to understand the point of the formula of the continuous FT. The formula of DFT comes from the formula of continual FT. Calculating the DFT coefficients of a signal makes the working of DFT more understandable.

The main goal of this article is to demonstrate how we can get to the teaching of 2D DFT from starting 1D continuous FT step by step. We represent some images and their FT to demonstrate 2D DFT. Of course, there are several images that can be presented to students to demonstrate other attributes of 2D DFT.

WT can also be studied after FT by pointing out the similarities and the differences. The idea and the image (see Figure 17) presented in this article make the working of WT more understandable.

\section{References}

[1] A. Ambrus, Bevezetés a matematika didaktikába, ELTE egyetemi jegyzet Eötvös Kiadó, 1995.

[2] T. Andreescu and D. Andrica, Complex Numbers from A to ... Z, Birkhäuser, 2006.

[3] I. N. Brohnstein, K. A. Semendyayan, G. Musiol and H. Muehling, Handbook of Mathematics, 5th Edition, Springer, 2007.

[4] Fourier transform, 2011, http://homepages.inf.ed.ac.uk/rbf/HIPR2/fourier.htm. 
[5] Fourier transform tutorial, 2011, http://www.fourier-series. com/f-transform/index.html.

[6] R. C. Gonzales and R. E. Woods, Digital Image Processing, Third Edition, Pearson Education Inc., 2008.

[7] R. M. Gray and L. D. Davisson, An Introduction to Statistical Signal Processing, Cambridge University Press, 2004.

[8] http://en.wikipedia.org/wiki/Analog_signal, 2011.

[9] Innovative teaching of Fourier series using Labview, 2011, http://dynsys . uml . edu/papers/ASEE_2006/ASEE2006_728_Fourier_Series_022706.pdf.

[10] Introduction to Fourier transforms for image processing, 2011, http://www.cs.unm. edu/ brayer/vision/fourier.html.

[11] L. Klingenberg, Frequency Domain Using Excel, 2010, http://userwww.sfsu.edu/ larryk/Common\%20Files/Excel.FFT. pdf.

[12] C. V. Loan, Computational Frameworks for the Fast Fourier Transform, Society for Industrial and Applied Mathematics, 1992.

[13] J. Gy. Obádovics, Matematika I., II., Scolar kiadó, Budapest, 1994.

[14] R. Polikar, The Wavelet Tutorial, 2011, http://users.rowan. edu/ polikar/WAVELETS/WTpart3.html.

[15] S. W. Smith, The Scientist and Engineer's Guide to Digital Signal Processing, 2nd Edition, California Technical Publishing, 2003.

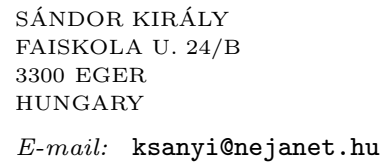

(Received December, 2010) 